ARTICLE

\title{
Tetradiketone macrocycle for divalent aluminium ion batteries
}

\author{
Dong-Joo Yoo (1) ${ }^{1}$, Martin Heeney (1) ${ }^{2}$, Florian Glöcklhofer (iD ${ }^{2 凶}$ \& Jang Wook Choi (1) ${ }^{1,3 凶}$
}

Contrary to early motivation, the majority of aluminium ion batteries developed to date do not utilise multivalent ion storage; rather, these batteries rely on monovalent complex ions for their main redox reaction. This limitation is somewhat frustrating because the innate advantages of metallic aluminium such as its low cost and high air stability cannot be fully taken advantage of. Here, we report a tetradiketone macrocycle as an aluminium ion battery cathode material that reversibly reacts with divalent $\left(\mathrm{AlCl}^{2+}\right)$ ions and consequently achieves a high specific capacity of $350 \mathrm{mAh} \mathrm{g}^{-1}$ along with a lifetime of 8000 cycles. The preferred storage of divalent ions over their competing monovalent counterparts can be explained by the relatively unstable discharge state when using monovalent $\mathrm{AlCl}_{2}{ }^{+}$ions, which exert a moderate resonance effect to stabilise the structure. This study opens an avenue to realise truly multivalent aluminium ion batteries based on organic active materials, by tuning the relative stability of discharged states with carrier ions of different valence states.

\footnotetext{
${ }^{1}$ School of Chemical and Biological Engineering and Institute of Chemical Processes, Seoul National University, Gwanak-Gu, Seoul, Republic of Korea.

${ }^{2}$ Department of Chemistry and Centre for Processable Electronics, Imperial College London, London, UK. ${ }^{3}$ Department of Materials Science and Engineering, Seoul National University, Gwanak-Gu, Seoul, Republic of Korea. ${ }^{凶}$ email: f.glocklhofer@imperial.ac.uk; jangwookchoi@snu.ac.kr
} 
S tate-of-the-art lithium-ion batteries (LIBs) are predominantly used as power sources for a variety of mobile electronic devices and electric vehicles. Although LIBs have made remarkable progress in terms of various aspects of battery performance, attention to so-called 'beyond LIBs' is incessantly growing, mainly because of concerns related to the unpredictable supply of raw materials employed in $\mathrm{LIBs}^{1,2}$. In particular, the uneven distribution and fluctuating price of lithium and transition metal precursors reflect these shortcomings ${ }^{3,4}$. Moreover, the innate safety risk associated with organic electrolyte-based LIBs has driven the on-going search for alternative safe battery systems. Among these candidates, aluminium (Al)-based batteries have recently received considerable attention because $\mathrm{Al}$ is the third most abundant element in the Earth's crust and, in its metallic form, it can deliver a high theoretical capacity of $2980 \mathrm{mAh} \mathrm{g}^{-1}$ or $8506 \mathrm{mAh} \mathrm{cm}^{-3}$ by taking advantage of the trivalent charge state $^{5-7}$. Metallic $\mathrm{Al}$ is also stable upon exposure to air and moisture, unlike its $\mathrm{Li}$ and $\mathrm{Na}$ metal counterparts. Nonetheless, the development of aluminium ion batteries (AIBs) has lagged behind at the research stage. One of the main hurdles to overcome is to develop cathode materials capable of storing multivalent Alcontaining carrier ions. In practice, finding such materials with sufficiently high ionic conductivity is a non-trivial matter, because the multi-valency of $\mathrm{Al}$ or $\mathrm{Al}$-complex ions induces strong Coulombic interaction with anionic frameworks ${ }^{8,9}$.

Ironically, most cathode materials identified to date in AIBs operate by storing monovalent $\mathrm{Al}$-complex ions, such as $\mathrm{AlCl}_{4}{ }^{-}$. These materials include carbon materials ${ }^{10-13}$, metal sulfides ${ }^{14-16}$ and metal selenides ${ }^{17,18}$. In the case of carbon materials, ever since the original report ${ }^{19}$ by Lin et al., who revealed the reversible storage of $\mathrm{AlCl}_{4}{ }^{-}$ions in graphite, various graphene-based materials were explored. However, this reaction, which exploits $\mathrm{AlCl}_{4}{ }^{-}$ions from the electrolyte, is unfavourable from an energy density consideration because the amount of electrolyte becomes a factor limiting the energy density. On the other hand, certain metal oxides $^{20-22}$ and sulfides ${ }^{23-25}$ exhibited reversible storage of trivalent $\mathrm{Al}$ ions $\left(\mathrm{Al}^{3+}\right)$. However, the strong Coulombic interaction between
$\mathrm{Al}^{3+}$ and the hosts makes facile ion diffusion difficult at reasonably high current densities. Rather, $\mathrm{Al}^{3+}$ ions could unwantedly transform oxides and sulfides to $\mathrm{Al}_{2} \mathrm{O}_{3}$ and $\mathrm{Al}_{2} \mathrm{~S}_{3}$ at the surface 26,27 . Notably, Chevrel-phase molybdenum sulfide $\left(\mathrm{Mo}_{6} \mathrm{~S}_{8}\right)$ with exceptionally large ionic channels is the only host material that has been proven to reversibly store $\mathrm{Al}^{3+}$ ions. However, the available specific capacity was only $100 \mathrm{mAh} \mathrm{g}^{-1}$ with a low operating voltage of $0.5 \mathrm{~V}$ even at the high temperature of $50{ }^{\circ} \mathrm{C}^{28,29}$.

The choice of materials for AIB cathodes was recently expanded to a family of organic molecules ${ }^{30-34}$ because the availability of sufficient intermolecular space as a result of the weak Van der Waals intermolecular interactions can be utilised for the diffusion and storage of the bulky aluminium complex carrier ions. These internal structures are also beneficial for releasing the strain generated during repeated insertion and extraction of the bulky complex ions, thereby achieving long-term cyclability. Despite these structural advantages, organic molecules are still not able to utilise the multi-valency of the $\mathrm{Al}$ ion for storage in their intermolecular space, thus they are adversely affected by the same limitation as the aforementioned series of inorganic materials.

Herein, we report a tetradiketone (TDK) macrocycle as a highcapacity cathode material for divalent AIBs. Particularly, the design we introduce exploits the radical destabilisation effect in the active molecule to preferentially induce divalent ion storage. A combination of density functional theory (DFT) calculations and experimental analyses reveal that adjacent carbonyl groups located at each corner of the TDK reversibly bind with a divalent $\mathrm{AlCl}^{2+}$ ion upon reduction, delivering an exceptionally high specific capacity of $350 \mathrm{mAh} \mathrm{g}^{-1}$ as an AIB cathode. Moreover, TDK exhibited excellent cyclability of $78 \%$ retention after 8000 cycles. This study offers useful insights into the way in which active molecules can be designed to activate their capability of storing divalent aluminium ions and thus take due advantage of multivalent batteries.

\section{Results}

Design and properties of TDK macrocycle. The TDK macrocycle was synthesised (Supplementary Fig. 1) by adapting and a<smiles>CC(C)(C)c1ccc(C(=O)C(=O)c2ccc(C(=O)C(=O)c3ccc(C(=O)C(=O)c4ccc(C(=O)C(=O)c5ccc(C(=O)C(=O)c6ccccc6)cc5)cc4)cc3)cc2)cc1</smiles>

b

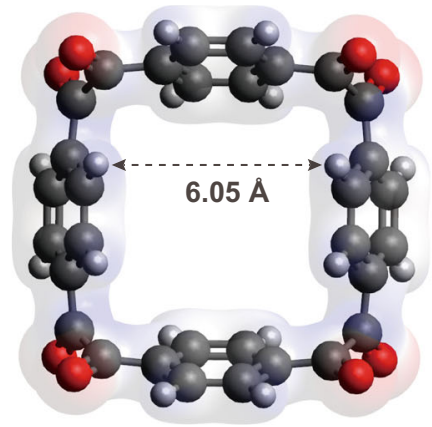

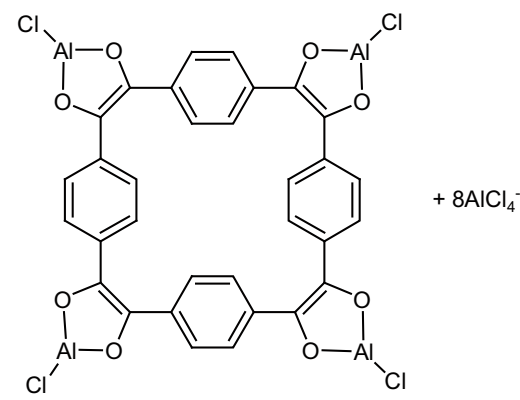

C

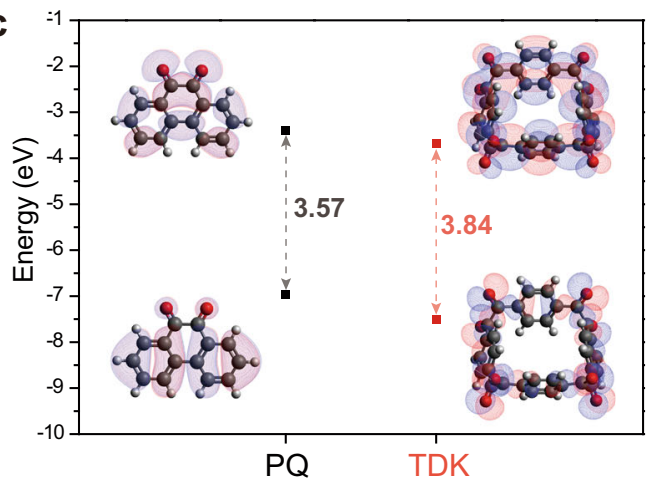

Fig. 1 Design and properties of the tetradiketone (TDK) macrocycle. a llustration of electrochemical redox mechanism of TDK. b Optimised structure and electron density of TDK in its pristine state. c HOMO-LUMO energy levels of the optimised structures of phenanthrenequinone (PQ) and TDK. 
combining the procedures developed by Miljanić and Bunz et al. ${ }^{35,36}$ to improve the purity of the target compound and double the overall yield. In the first step of the synthesis, cyclotetrabenzoin was produced as an intermediate, which was isolated by filtration for use in the next step, that is, its oxidation to TDK in concentrated $\mathrm{HNO}_{3}$. The crude product was obtained in solid form by filtration. Subsequent Soxhlet extraction with chloroform selectively dissolved TDK and separated it from insoluble side products. The structure and purity of the final product was confirmed by a combination of nuclear magnetic resonance (NMR), Fourier-transform infra-red (FT-IR) and thermogravimetric analysis (TGA) (Supplementary Figs. 2 and 3).

The reaction scheme of TDK with $\mathrm{AlCl}_{3}$ is presented in Fig. 1a. From the perspective of its chemical structure, TDK can be reduced (or discharged) by accepting as many as $8 \mathrm{e}^{-}$in such a way that the adjacent carbonyl groups at each corner form a chelate with one $\mathrm{AlCl}^{2+}$ ion. The reverse reaction occurs during oxidation (or charging). The benzene rings in TDK are not equiplanar with the macrocyclic plane owing to their rotational freedom, and a large void of $6.05 \AA$ is present in the centre of the macrocycle (Fig. 1b). This void could possibly facilitate efficient transport of the charge carrier ions. Note that this molecular structure of TDK is in contrast with that of phenanthrenequinone (PQ), another representative molecule that would be suitable as an AIB cathode material, in that PQ has a planar structure comprising a series of conjugated bonds without rotational freedom (see structure in Fig. 1c, inset). The DFT calculations revealed that, despite the disconnected conjugated bonds in the macrocycle, the energy difference between the highest occupied molecular orbital (HOMO) and lowest unoccupied molecular orbital (LUMO) of TDK is $3.84 \mathrm{eV}$, which closely approximates the $3.57 \mathrm{eV}$ of PQ (Fig. 1c).

Reaction mechanism of TDK with divalent aluminium complex ions. To elucidate the reaction mechanism of TDK in comparison with $\mathrm{PQ}$, we conducted DFT calculations to estimate the formation energies of both molecules when each molecule binds with a monovalent $\left(\mathrm{AlCl}_{2}{ }^{+}\right)$or divalent $\left(\mathrm{AlCl}^{2+}\right)$ complex ion. All the structures in Fig. 2a, b are structurally optimised states (details of the calculation appear in the Method section). As the formation energy refers to thermodynamic stability, the formation energies of the discharged states can uncover the preferred charge carriers for the electrochemical reaction in electrolytes in which $\mathrm{Al}_{2} \mathrm{Cl}_{7}-$ can yield various forms of aluminium complex ions such as $\mathrm{AlCl}_{4}{ }^{-}, \mathrm{AlCl}_{2}{ }^{+}, \mathrm{AlCl}^{2+}$ and $\mathrm{Al}^{3+}$. In the case of $\mathrm{PQ}$ and TDK, the adjacent carbonyl groups possibly chelate $\mathrm{AlCl}_{2}+$ or $\mathrm{AlCl}^{2+}$ ions upon reduction. Therefore, the energy difference between the states bonded with these two carrier ions can be taken as a descriptor for indicating the major carrier ion.

In the case of $\mathrm{PQ}$, only one possible stable configuration exists upon binding with each carrier ion (Fig. 2a), denoted as PQ- $\mathrm{AlCl}_{2}$ and $\mathrm{PQ}-\mathrm{AlCl}$, respectively. The formation energies of $\mathrm{PQ}-\mathrm{AlCl}_{2}$ and $\mathrm{PQ}-\mathrm{AlCl}$ were -9.436 and $-9.459 \mathrm{eV}$, respectively, implying that $\mathrm{PQ}-\mathrm{AlCl}$ is more thermodynamically stable. However, considering that the energy difference between the two complex states is merely $0.023 \mathrm{eV}$, the $\mathrm{PQ}-\mathrm{AlCl}_{2}$ form cannot be ruled out in reality. Taking ionic diffusion into account, binding with $\mathrm{AlCl}_{2}{ }^{+}$ions could be overall preferred, as was indeed experimentally verified ${ }^{32,37}$. On the other hand, in the case of TDK, a total of eight possible states can be postulated: four with $\mathrm{AlCl}_{2}{ }^{+}$ ions and the other four with $\mathrm{AlCl}^{2+}$ ions. To note, although there are two possible configurations for TDK- $2 \mathrm{AlCl}_{2}$ and TDK-2AlCl in which the carrier ions are located in the adjacent and opposite positions, the configurations with lower formation energy are presented. The average formation energies of TDK- $n \mathrm{AlCl}_{2}$ and TDK- $n \mathrm{AlCl}$ were calculated to be -8.939 and $-9.318 \mathrm{eV}$, respectively, implying that binding with $\mathrm{AlCl}^{2+}$ ions is thermodynamically more favourable. Furthermore, the energy difference between TDK- $n \mathrm{AlCl}_{2}$ and TDK- $n \mathrm{AlCl}$ was found to be $0.379 \mathrm{eV}$, which is beyond the range of the overpotential in typical electrochemical measurements. This suggests that TDK prefers divalent over monovalent ion storage.

Importantly, the preferential binding of TDK with the $\mathrm{AlCl}^{2+}$ ion can be understood in relation to its relative instability upon binding with the $\mathrm{AlCl}_{2}{ }^{+}$ion when compared with PQ. It is worth noting that a quinone molecule normally generates a radical upon reduction with one electron (and binding with a monovalent ion), and the radical is stabilised by the delocalisation with the adjacent aromatic rings. From the viewpoint of the chemical structure, TDK has a lower benzene ring-to-carbonyl group ratio of 0.5 compared to 1.0 of $\mathrm{PQ}$, resulting in a less pronounced stabilisation of radicals by the resonance effect. This equally means that upon the formation of a radical, TDK can form a smaller number of resonance structures than PQ. This resonance effect was also revealed by atomic charge analysis as shown in Fig. 2c. In the pristine state, the oxygen atoms of PQ and TDK have a similar atomic charge of -0.5502 and -0.5429 on average, respectively (Supplementary Fig. 4). When an $\mathrm{AlCl}_{2}^{+}$ion is bound, the oxygen atoms of PQ each have an atomic charge of -0.3297 , whereas that of TDK is clearly smaller at -0.3049 , indicating weaker binding with the $\mathrm{AlCl}_{2}+$ ion. This weaker binding of TDK with $\mathrm{AlCl}_{2}{ }^{+}$is once again associated with the less prominent stabilisation of radicals by the delocalisation. By contrast, when an $\mathrm{AlCl}^{2+}$ ion is bound, the oxygen atoms of PQ and TDK have a similar atomic charge of -0.9045 and -0.9031 , respectively, indicating that the binding strength with the $\mathrm{AlCl}^{2+}$ ion is similar for both molecules. Therefore, the difference in binding strength between the $\mathrm{AlCl}_{2}{ }^{+}$and $\mathrm{AlCl}^{2+}$ ions is greater for TDK, and serves as the origin of its preferential binding with the $\mathrm{AlCl}^{2+}$ ion.

Ex situ characterisation of TDK in the discharged state. Figure 3 a shows the charge-discharge voltage profile of TDK when a current density of $20 \mathrm{~mA} \mathrm{~g}^{-1}$ was applied. TDK showed a plateau at approximately $1.3 \mathrm{~V}$ vs. $\mathrm{Al} / \mathrm{Al}^{3+}$ which is similar to that of $\mathrm{PQ}$ $\left(1.4 \mathrm{~V}\right.$ vs. $\left.\mathrm{Al} / \mathrm{Al}^{3+}\right)$. The reversible capacity of TDK at this current density was $350 \mathrm{mAh} \mathrm{g}^{-1}$, which corresponds to the storage of $7 \mathrm{e}^{-}$per molecular unit. This capacity is far beyond $203 \mathrm{mAh} \mathrm{g}^{-1}$, which corresponds to the storage of $4 \mathrm{e}^{-}$when all of the carbonyl groups of TDK are utilised to bind with monovalent ions. This strongly hints that the storage mechanism dominantly involves divalent ions. Remarkably, the specific capacity of TDK is also far above those of other materials reported as AIB cathodes to date, revealing the beneficial effect of divalent ion storage.

We monitored the change in functional groups during discharging by carrying out ex situ FT-IR spectroscopy and compared the results with the calculated IR vibration peaks. The FT-IR measurements of TDK were conducted after the second cycle at a current density of $20 \mathrm{~mA} \mathrm{~g}^{-1}$. Regarding the calculated peaks, we first confirmed their reliability by checking that the main peaks of TDK were in good agreement with the calculated ones (Supplementary Fig. 3a). Based on the confirmed reliability, we characterised the TDK electrodes at the different discharged states marked in Fig. 3a. These measurement points were set to enable the states of TDK to be captured after binding with every $\mathrm{AlCl}^{2+}$ ion. Based on this analysis, the following five features are noticeable from the viewpoint of both the simulation (Fig. 3b) and the experiments (Fig. $3 \mathrm{c}$ ): (1) The peak intensity of the $\mathrm{C}=\mathrm{O}$ asymmetric stretching modes (Supplementary Video 1) of TDK at 

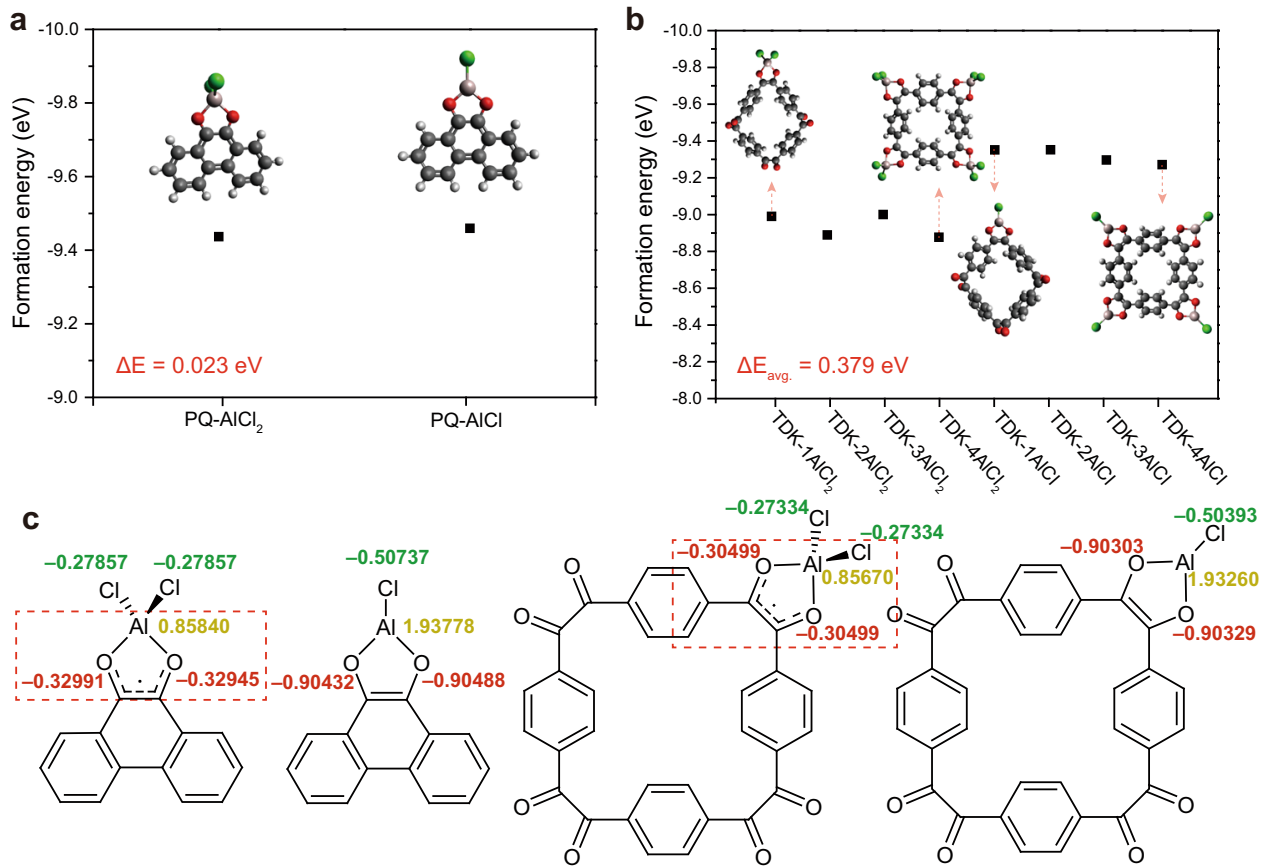

Fig. 2 Thermodynamic stability of $\mathbf{P Q}$ and TDK when bound with monovalent $\left(\mathrm{AICl}_{\mathbf{2}}{ }^{+}\right)$or divalent $\left(\mathbf{A I C l}{ }^{2+}\right)$ carrier ions. a, b Formation energies of a $\mathrm{PQ}$ and $\mathbf{b}$ TDK when bound with $\mathrm{AlCl}_{2}+$ or $\mathrm{AlCl}^{2+}$ complex ions. The four different states correspond to the cases when different numbers of $\mathrm{AICl}_{2}+$ or $\mathrm{AlCl}^{2+}$ ions are bound. The optimised molecular structures of selected TDK-nAICl 2 or TDK-nAICl combinations are presented. $\mathbf{c}$ Atomic charge analysis of $\mathrm{PQ}$ and TDK when bonded to a single $\mathrm{AlCl}_{2}+$ or $\mathrm{AICl}^{2+}$ ion.
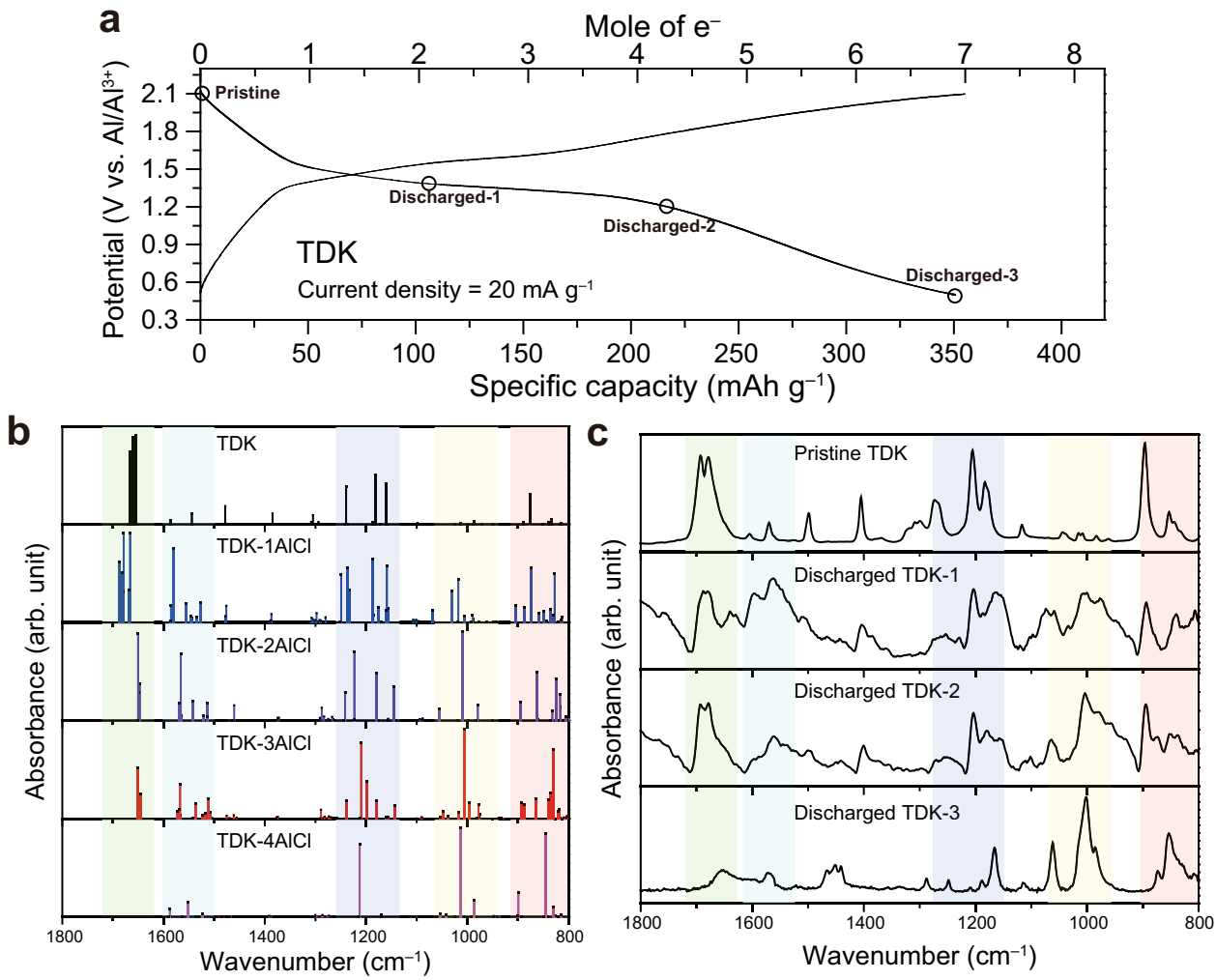

Fig. 3 Ex situ FT-IR characterisation of TDK. a Galvanostatic voltage profiles of TDK at the current density of $20 \mathrm{~mA} \mathrm{~g}^{-1}$. $\mathbf{b}$ Calculated IR vibration peaks of TDK when bound with $\mathrm{AICl}^{2+}$ ions. c Measured FT-IR absorbance spectra of the TDK electrode. The discharging points at which the measurements were carried out are indicated in (a). 
$1686 \mathrm{~cm}^{-1}$ gradually decreased as a result of binding with an increasing number of $\mathrm{AlCl}^{2+}$ ions (green shades in Fig. 3b, c). (2) The peaks of the $\mathrm{C}-\mathrm{C}$ and $\mathrm{C}=\mathrm{C}$ stretching modes near the $\mathrm{C}=\mathrm{O}$ that binds with the $\mathrm{AlCl}^{2+}$ ion (Supplementary Videos 2 and 3) were observed at 1581 and $1527 \mathrm{~cm}^{-1}$ for TDK-1AlCl, but gradually disappeared as more carbonyl groups formed bonds with $\mathrm{AlCl}^{2+}$ ions (cyan shades in Fig. 3b, c). (3) The peak of the $\mathrm{C}-\mathrm{C}$ and $\mathrm{C}=\mathrm{C}$ stretching modes (Supplementary Video 4) of TDK at $1180 \mathrm{~cm}^{-1}$ was shifted to $1210 \mathrm{~cm}^{-1}$ in the case of TDK4AlCl (Supplementary Video 5) (navy shades in Fig. 3b, c). (4) The peak at $1013 \mathrm{~cm}^{-1}$ corresponding to the $\mathrm{C}-\mathrm{C}$ and $\mathrm{C}=\mathrm{C}$ stretching modes and $\mathrm{C}=\mathrm{O}$ stretching modes over the entire macrocycle appeared (Supplementary Video 6) and grew (Supplementary Video 7) to become the main peak of TDK4AlCl (yellow shades in Fig. 3b, c). (5) The peak of the C-H wagging and $\mathrm{C}=\mathrm{O}$ asymmetric stretching modes (Supplementary Video 8) of TDK at $880 \mathrm{~cm}^{-1}$ was replaced by the peak of TDK$4 \mathrm{AlCl}$ at $844 \mathrm{~cm}^{-1}$, corresponding to its $\mathrm{C}-\mathrm{H}$ wagging and $\mathrm{O}-\mathrm{Al}$ stretching modes (Supplementary Video 9) (red shades in Fig. 3b, c). For all of these observations, the calculations and actual analyses corresponded well indeed. The main peaks of discharged TDK were preserved after 5 cycles, indicating the robust nature of the reaction with $\mathrm{AlCl}^{2+}$ ions (Supplementary Fig. 5b). In addition, the calculated IR vibration peaks of TDK upon binding with $\mathrm{AlCl}_{2}+$ ions were conspicuously mismatched with the experimental ones (Supplementary Fig. 6), further supporting that the storage mechanism of TDK is based on $\mathrm{AlCl}^{2+}$ ions.

Electrochemical performance of aluminium batteries. To minimise the dissolution of active molecules into the electrolyte, we infiltrated TDK into the pores of activated carbon (AC) by simply replacing carbon black with $\mathrm{AC}$ as the conductive agent. This choice of conductive agent was synergetic with the high solubility of TDK in $N$-methyl-2-pyrrolidone (NMP), a slurry solvent commonly used in battery fabrication, such that TDK naturally penetrated the pores of AC while the slurry was being mixed and dried. We first indirectly recognised the infusion of TDK into the pores of AC by the fact that the original yellowish colour of TDK powder was not observed after the evaporation of NMP (Supplementary Fig. 7). Scanning electron microscopy (SEM) provided more direct verification of the impregnation of TDK. The morphology of $\mathrm{AC}$ was restored in the case of the TDK-AC blend after the evaporation of NMP, whereas TDK was found to be recrystallised separately when blended with nonporous carbon materials such as Super P and multiwalled carbon nanotubes (MWCNTs) (Supplementary Fig. 8).

Further evidence that TDK had penetrated the pores of AC was obtained by recording the adsorption-desorption isotherms using $\mathrm{N}_{2}$ gas (Supplementary Fig. 9). Unlike the TDK-Super $\mathrm{P}$ and TDK-MWCNT blends, the specific surface area (SSA) of the TDK-AC blend $\left(705 \mathrm{~m}^{2} \mathrm{~g}^{-1}\right)$ was significantly smaller than that of pristine AC $\left(2347 \mathrm{~m}^{2} \mathrm{~g}^{-1}\right)$ as a result of the pores being filled with TDK. The infiltration of TDK was additionally detected by $\mathrm{X}$-ray diffraction (XRD) analysis (Supplementary Fig. 10). Although the characteristic XRD peaks were observed for the TDK-AC blend, their intensities were weakened owing to the confining effect in the pores. When a TDK solution is condensed by allowing the solvent to evaporate, TDK alone tends to crystallise as indicated by its XRD peaks (Supplementary Fig. 10). However, when confined within a space with comparable dimensions to that of TDK clusters, molecule-to-wall interactions could perturb intermolecular interaction and therefore the crystallinity of TDK. The confinement effect was much less significant with the TDK-Super P and TDK-MWCNT blends because these carbon materials do not form pores with such small dimensions. Note that low crystallinity is usually beneficial as it enables active molecules to sustain themselves against dissolution in the electrolyte during cycling; by contrast, molecules with high crystallinity are more vulnerable to solvation by the electrolyte solvent ${ }^{38,39}$.

The electrochemical performance of TDK was evaluated by various electrochemical analyses (Fig. 4). It is noteworthy that AC alone exhibited a low specific capacity of $40 \mathrm{mAh} \mathrm{g}^{-1}$, implying that its contribution to the capacity of TDK-AC blend is not substantial (Supplementary Fig. 11). To this end, TDK-Super P, MWCNT, or AC blends were subjected to galvanostatic cycling tests (Fig. 4a). All of the TDK-carbon blends showed similar specific capacities in their first two cycles (Supplementary Fig. 12). The application of a current density of $0.1 \mathrm{Ag}^{-1}$ resulted in the rapid decay of the capacities of the TDK-Super P and MWCNT blends to 55 and $90 \mathrm{mAh} \mathrm{g}^{-1}$ after the first 50 cycles, respectively, due to the severe dissolution problem. In the case of the TDK-AC blend, although it showed a capacity decrease from 226 to $170 \mathrm{mAh} \mathrm{g}^{-1}$ during the first 50 cycles, which probably originated from the dissolution of a small portion of recrystallised TDK on the surface of $\mathrm{AC}$ as detected in the XRD results (Supplementary Fig. 10), it retained a remarkably high capacity of $170 \mathrm{mAh} \mathrm{g}^{-1}$ after 300 cycles, revealing the confined TDK to be highly stable. To note, the capacity decay at the very low current density was mainly ascribed to irreversible oxidation of the electrolyte at high voltages in addition to the inevitable dissolution of discharged TDK (Supplementary Fig. 13). The superior performance of the TDK-AC blend was also observed in rate capability tests (Fig. 4b). An increase in the current density from 0.1 to $0.2,0.5,1$, and $2 \mathrm{~A} \mathrm{~g}^{-1}$ maintained the capacity from 185 to $145,111,90$ and $66 \mathrm{mAh} \mathrm{g}^{-1}$, respectively. When the current density was returned to $0.1 \mathrm{~A} \mathrm{~g}^{-1}$, the capacity of $165 \mathrm{mAh} \mathrm{g}^{-1}$ was recovered, showing the sustainable nature of the TDK-AC blend against a sweep across various current densities. The cycling performance of the TDK-AC blend was also stable such that when cycled at $1 \mathrm{~A} \mathrm{~g}^{-1}$, the cell preserved $78.0 \%$ of its original capacity even after 8000 cycles (Fig. 4c and Supplementary Fig. 14). The initial capacity increase at the high current density was attributed to an activation process required for electrolyte infiltration, which is supported by the charge transfer resistance $\left(R_{\mathrm{ct}}\right)$ that decreased during initial cycles (Supplementary Fig. 15).

The charge storage mechanism of electrode materials can be elucidated by scan rate-dependent cyclic voltammetry (CV) measurement, in which the capacity contribution is divided into faradaic and non-faradaic processes. The faradic process relates to a (de)intercalation and charge-transfer process, and the nonfaradaic process relates to capacitive ion adsorption in the electric double layer ${ }^{40,41}$. The contributions from these two processes can be identified by analysing the $\mathrm{CV}$ profiles at various scan rates according to the following relation between the peak current $(I)$ and the scan rate $(v)$

$$
I=a v^{b}
$$

in which the value of $b$ can be determined by the slope of the $(\log v)-(\log I)$ plot. Recall that $b=0.5$ corresponds to a diffusioncontrolled process, whereas $b=1$ exclusively indicates a capacitive-limited process. Using the redox peaks in the CV profiles (Supplementary Fig. 16a), we plotted the $(\log v)-\left(\log I_{p}\right)$ of the TDK-AC electrode (Fig. $4 \mathrm{~d}$ ). The $b$-values of the reduction and oxidation were 0.64 and 0.62 , respectively, implying that a large portion of charge storage is diffusion-controlled and that the diffusion kinetics of $\mathrm{AlCl}^{2+}$ ions in TDK may not be very efficient.

Effect of the valence state of the carrier ion on the electrochemical performance. The extent to which the kinetics 
a
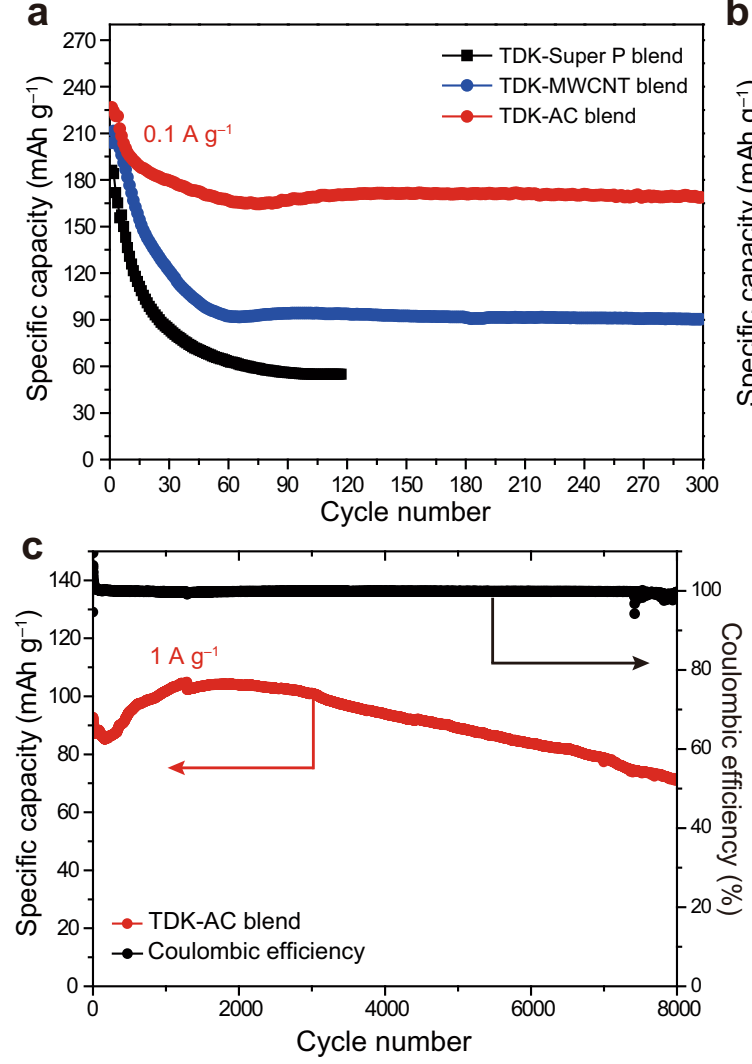

b
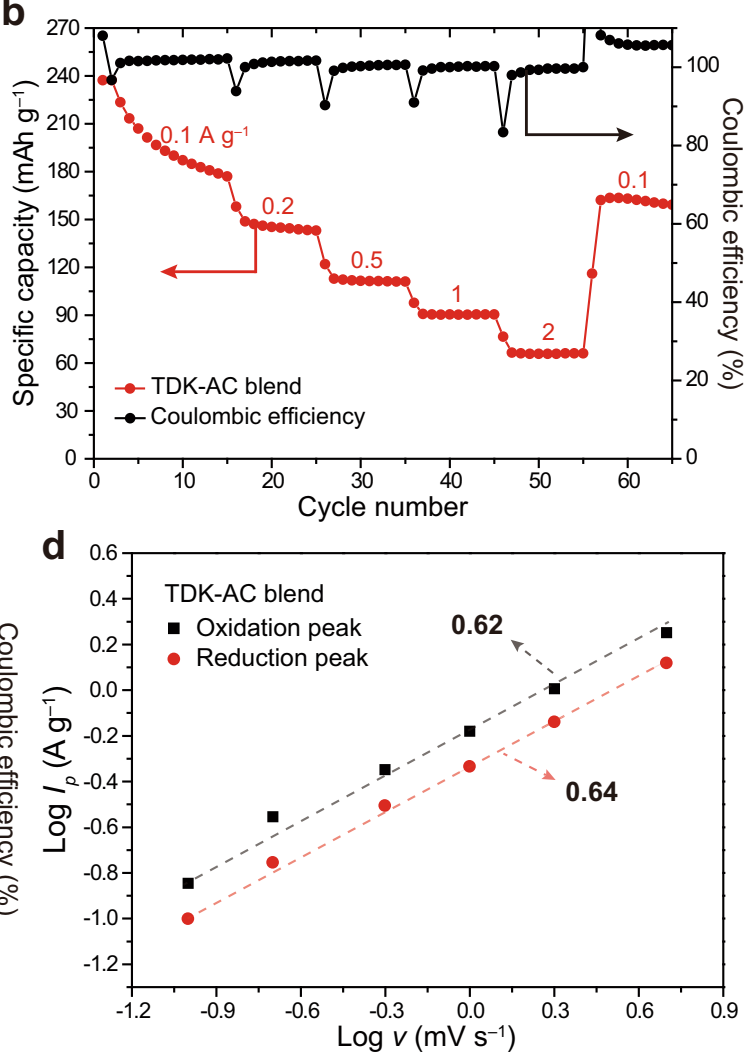

Fig. 4 Electrochemical performance of TDK. a Cycling performance of TDK with different carbon materials. $\mathbf{b}$ Rate capability of TDK-AC blend. $\mathbf{c}$ Longterm cycling performance of TDK-AC blend at a current density of $1 \mathrm{Ag}^{-1} \cdot \mathbf{d} \log v$-log $I_{p}$ plots to extract the $b$-values based on the relation $I_{p}=a v^{b}$.

depended on the charge carrier ions was determined by preparing graphite and PQ electrodes, which are known ${ }^{19,37}$ to exploit $\mathrm{AlCl}_{4}{ }^{-}$and $\mathrm{AlCl}_{2}{ }^{+}$ions for charge storage, respectively. The $b-$ values of graphite and PQ were extracted from the redox peaks in the CV profiles (Supplementary Fig. 16b, c). The $b$-values related to the reduction and oxidation of graphite were 0.92 and 0.91 , respectively, whereas those of PQ were 0.72 and 0.87 , respectively (Supplementary Fig. 17). Although both graphite and PQ utilise monovalent ions as carrier ions, the higher $b$-values and diffusion kinetics of graphite are attributed to its intrinsically high electrical conductivity. The fact that both graphite and PQ have $b$-values higher than those of TDK supports our findings that TDK stores divalent ions $\left(\mathrm{AlCl}^{2+}\right)$ and explains its relatively sluggish diffusion.

The effect of the functional groups on the charge carrier ions was examined by X-ray photoelectron spectroscopy (XPS) to analyse the graphite, $\mathrm{PQ}$, and TDK electrodes. Because the binding energy of the core electrons is sensitive to the chemical environment of the corresponding element, the XPS peak shift allows us to clarify the binding structures of the charge carrier ions. To note, all the XPS peaks were referenced with respect to the $\mathrm{C}-\mathrm{C}$ bond at $284.8 \mathrm{eV}$ in the $\mathrm{C} 1 s$ branch (Supplementary Fig. 18d). Prior to analysing the binding structures, we first verified that the charge carrier ions stored in the graphite, $P Q$, and $\mathrm{TDK}$ were $\mathrm{AlCl}_{4}{ }^{-}, \mathrm{AlCl}_{2}{ }^{+}$and $\mathrm{AlCl}^{2+}$ based on the fact that the atomic ratios of $\mathrm{Cl}$ to $\mathrm{Al}$ were $3.93,1.75$ and 0.97 , respectively (Supplementary Fig. 18). The atomic ratio in TDK-AlCl was further confirmed by energy dispersive spectroscopy mapping analysis (Supplementary Fig. 19). In the $\mathrm{Al} 2 p$ branches (Fig. 5a), the peaks at $74.1,74.8$ and $74.6 \mathrm{eV}$ were assigned to graphite$\mathrm{AlCl}_{4}, \mathrm{PQ}-\mathrm{AlCl}_{2}$ and $\mathrm{TDK}-\mathrm{AlCl}$, respectively. It is instructive to note that the binding energy shifts to a higher value when the element is bonded with functional groups or elements with higher electronegativity. On the basis of this rationale, the location of the $\mathrm{Al}$ peak of $\mathrm{PQ}-\mathrm{AlCl}_{2}$ at higher binding energy compared to that of graphite- $\mathrm{AlCl}_{4}$ can be understood by taking into account that $\mathrm{Al}$ is bonded with the electronegative carbonyl groups of PQ. Based on the same logic, the slightly lower binding energy of the $\mathrm{Al}$ peak of TDK-AlCl compared with that of PQ- $\mathrm{AlCl}_{2}$ can be explained by the fact that the $\mathrm{Al}$ in $\mathrm{TDK}-\mathrm{AlCl}$ is bonded with a single $\mathrm{Cl}$ atom. On the other hand, in the $\mathrm{Cl} 2 p$ branches (Fig. 5b), the peaks at $198.9 \mathrm{eV}$ were shared by $\mathrm{PQ}-\mathrm{AlCl}_{2}$ and $\mathrm{TDK}-\mathrm{AlCl}$, as the $\mathrm{Cl}$ atoms in $\mathrm{PQ}-\mathrm{AlCl}_{2}$ and $\mathrm{TDK}-\mathrm{AlCl}$ are both bound solely to the $\mathrm{Al}$ atom. In the case of graphite- $\mathrm{AlCl}_{4}$, however, the peak of $\mathrm{Cl}$ shifted downward to $197.9 \mathrm{eV}$ because $\mathrm{AlCl}_{4}$ interacts with the graphitic layers to a certain degree.

The energy density of electrode materials is by and large determined by their operating voltage and specific capacities. Even though this metric is generally valid, the energy density of an AIB should be carefully evaluated. In an AIB cell, the charge carrier ions used in the anode and cathode are different, which implies that at least one carrier ion must be supplied by the electrolyte and the amount of electrolyte should certainly be taken into consideration in the energy density calculation. Figure $5 \mathrm{c}$ shows the charge-discharge voltage profiles of the graphite, PQ and TDK cells. When considering only the operating voltage and specific capacity, the graphite, PQ and TDK cells deliver energy densities of 170,168 and $278 \mathrm{Wh} \mathrm{kg}^{-1}$, respectively. However, when the amounts of electrolyte are taken into account, the energy densities are changed as marked in Fig. 5d. In this sense, the charge carrier ion plays a role as the $\mathrm{Al}$-to- $\mathrm{Cl}$ ratio determines the amount of the electrolyte. Details of the calculation are given in the Supplementary Note. Although the rate performance of the graphite and PQ cells was higher as they take advantage of the 

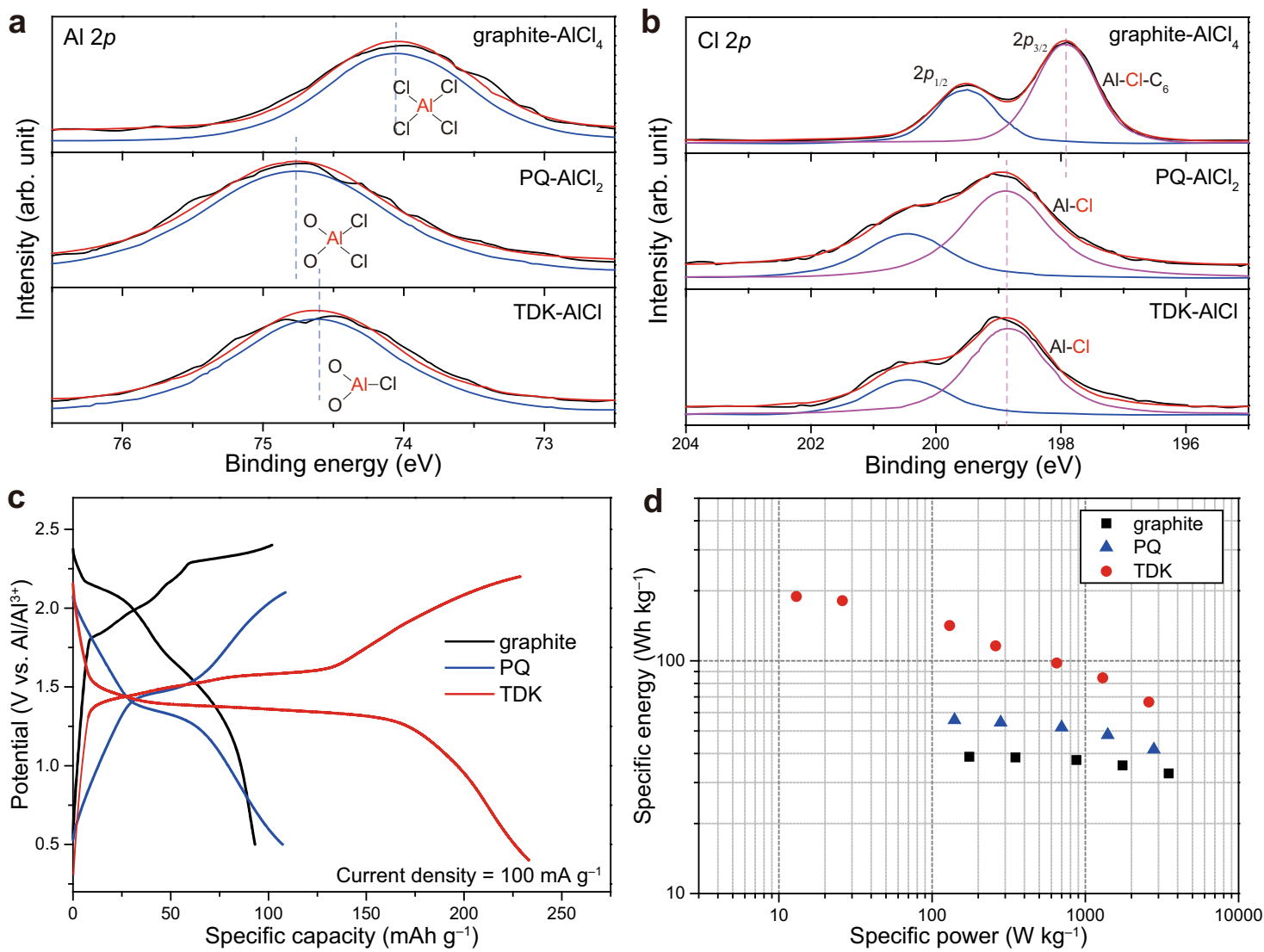

Fig. 5 Comparison of cathode materials exploiting $\mathbf{A I C l}_{\mathbf{4}}{ }^{-}, \mathbf{A l C l}_{\mathbf{2}}{ }^{+}$and $\mathbf{A I C l}{ }^{2}+$ ions. a, b XPS curves of graphite-AICl${ }_{4}, \mathrm{PQ}-\mathrm{AICl}{ }_{2}$ and $\mathrm{TDK}-\mathrm{AICl}$; a $\mathrm{Al} 2 p$ and $\mathbf{b} \mathrm{Cl} 2 p$ branches. $\mathbf{c}$ Galvanostatic voltage profiles of graphite, PQ and TDK when the current density is $100 \mathrm{~mA}^{-1}$. d Ragone plots of cells based on graphite, $\mathrm{PQ}$ and TDK. The required amount of electrolyte was taken into account for this calculation.

more facile diffusion of the carrier ions (Supplementary Fig. 20), their energy densities are only 38 and $55 \mathrm{Wh} \mathrm{kg}^{-1}$, respectively. On the contrary, the TDK cell exhibited far higher energy density of $189 \mathrm{Wh} \mathrm{kg}^{-1}$ and power density of $2600 \mathrm{~W} \mathrm{~kg}^{-1}$ by utilising the divalent $\mathrm{AlCl}^{2+}$ ions and this performance is linked to the aforementioned superior specific capacity of the TDK electrode. Although the specific energy values would need to be further improved to attain practical competitiveness, the present study demonstrates the impact of exploiting multivalent carrier ions and the relevance of active material design.

\section{Discussion}

The core advantage of AIBs is its capability to store multivalent carrier ions to increase the specific capacity of electrode materials. Unfortunately, nearly all the cathode materials reported to date operate on the basis of the storage of monovalent complex ions such that the true benefit of AIBs has not been fully taken advantage of. We introduced the 'relative stability' of discharged states bearing carrier ions with different valence states as a key parameter to determine the principal carrier ion for active diketone molecules in AIBs. The structural stability of the discharged state with monovalent ions is particularly determined by the resonance effect of their benzene rings to stabilise the radical state. As predicted by the low ring-to-carbonyl group ratio, the TDK radical, upon binding with the monovalent ions, experiences moderate resonance stabilisation, rendering divalent ion storage dominant. Based on this design rationale toward achieving divalent ion storage, TDK delivers an extraordinarily high specific capacity of $350 \mathrm{mAh} \mathrm{g}^{-1}$ as an AIB cathode material with an operating voltage of $1.3 \mathrm{~V}\left(\mathrm{Al} / \mathrm{Al}^{3+}\right)$ as well as a lifetime that exceeds 8000 cycles. In a greater context, our study suggests ways in which to design diketone molecules to activate the multivalent ion storage mechanism; that is, to destabilise the radical formed upon reaction with monovalent ions.

\section{Methods}

Material preparation. Terephthalaldehyde $(20.1 \mathrm{~g}, 150 \mathrm{mmol})$, water $(450 \mathrm{~mL})$ and 2-methoxyethanol $(450 \mathrm{~mL})$ were added to a $1 \mathrm{~L}$ round-bottom flask equipped with a reflux condenser. The resulting stirred suspension was purged with nitrogen and heated to reflux under nitrogen to dissolve the starting material. $\mathrm{NaCN}$ (735 mg, $15.0 \mathrm{mmol}$ ) was then added and the reaction mixture was maintained under reflux for 3 days. The precipitated cyclotetrabenzoin intermediate was isolated by filtration while the reaction was still hot (using a preheated sintered glass funnel) and was then washed several times with water, methanol, and diethyl ether. After drying in vacuo, $10.1 \mathrm{~g}(18.8 \mathrm{mmol})$ of the crude intermediate was obtained, and this was placed in a $1 \mathrm{~L}$ round-bottom flask and suspended in concentrated $\mathrm{HNO}_{3}(70 \%, 380 \mathrm{~mL})$. The flask was equipped with a reflux condenser and the reaction was slowly heated to reflux, resulting in the initial intense formation of nitrous fumes. After $60 \mathrm{~h}$ at reflux, the reaction was cooled to room temperature and water $(380 \mathrm{~mL})$ was added carefully. The solid was isolated by filtration, washed twice with water, ethanol, and diethyl ether, and extracted with chloroform in a Soxhlet extractor for 3 days to dissolve the TDK and separate it from insoluble side products. Evaporation of the chloroform yielded pure TDK $(5.24 \mathrm{~g}, 9.92$ $\mathrm{mmol}$ ) as a bright yellow powder in an overall yield of $26 \% .{ }^{1} \mathrm{H}$ NMR (400 MHz, DMSO- $\left.\mathrm{d}_{6}\right): \delta=7.90(\mathrm{~s}, 16 \mathrm{H}) \mathrm{ppm}$; in accordance with the literature ${ }^{36} .{ }^{13} \mathrm{C} \mathrm{NMR}$ $\left(101 \mathrm{MHz}, \mathrm{DMSO}-d_{6}\right): \delta=194.5,135.8,130.5 \mathrm{ppm}$.

Cell preparation and electrochemical measurements. The electrodes were prepared using the following procedures. TDK, the conductive agent, and poly (vinylidene difluoride) (PVDF, Arkema) binder were dispersed in $\mathrm{N}$-methyl 2pyrrolidone (NMP) in a weight ratio of 50:40:10. The slurry was cast onto tantalum foil (99.95\%, Thermo Fisher) by using the doctor blade technique, and the cast electrodes were dried under vacuum at $80^{\circ} \mathrm{C}$ for $24 \mathrm{~h}$. The conductive agents were Super P, MWCNTs or AC (AC0830, Asahi Organic Chemicals). The mass loading of TDK in each electrode was $1.5 \mathrm{mg} \mathrm{cm}^{-2}$. For the PQ electrodes, PQ, AC and PVDF binder were employed in a weight ratio of 50:40:10. For the graphite 
electrodes, graphite (N006, Digichem), Super P and PVDF binder were used in a weight ratio of 80:10:10. The aluminium electrolyte was prepared by slowly adding aluminium trichloride $\left(\mathrm{AlCl}_{3}\right)$ to ethyl-3-methylimidazolium chloride (EMImCl) in a molar ratio of 1.5:1 ( $\left.\mathrm{AlCl}_{3}: \mathrm{EMImCl}\right)$. The electrochemical measurements were conducted by using modified Swagelok-type cells in which a stainless-steel rod and a glassy carbon rod were used as current collectors for the anode and cathode, respectively. The cells were composed of an aluminium metal foil anode, a glass fibre membrane (GF/D Whatman) and a cathode. The entire cell was assembled inside an argon-filled glove box. Galvanostatic measurements and cyclic voltammetry were performed using a battery cycler (WBCS3000L, Wonatech) at $25^{\circ} \mathrm{C}$ The Coulombic efficiency was defined as the ratio of charge capacity to discharge capacity because each cell began to operate by discharging.

Characterisation. TDK was analysed by NMR spectroscopy and TGA to verify the functional groups and purity. Ex situ characterisations were conducted by analysing the electrode samples by FT-IR (JASCO FT/IR-6700). The morphologies of the TDK electrodes comprising various carbon materials were characterised by using field-emission scanning electron microscopy (JSM-7800F Prime, JEOL) on the instrument housed at the National Center for Inter-University Research Facilities (NCIRF) at Seoul National University. The SSAs of TDK electrodes with various carbon materials were obtained from $\mathrm{N}_{2}$ adsorption-desorption measurements by using a porosity analyser (Micromeritics, 3FLEX) operating at $87 \mathrm{~K}$. The $\mathrm{XRD}$ profiles were obtained by using an X-ray diffractometer (SmartLab, Rigaku) based on $\mathrm{Cu}-\mathrm{Ka}(\lambda=0.15406 \mathrm{~nm})$ radiation. The chemical binding states of discharged graphite, PQ and TDK were characterised by XPS (Sigma Probe, Thermo VG Scientific) with an Mg Ka line as the X-ray source. All the electrode samples for ex situ characterisations were washed with 1,2-dichloroethane in an argon-filled glove box, followed by drying under vacuum for $3 \mathrm{~h}$.

DFT calculations. Geometrical optimisations, energy calculations and atomic charge analysis were carried out without symmetry restriction using the B3LYP hybrid density functional implemented in the GAUSSIAN 09 software package ${ }^{42}$. The $6-31+G(d)$ basis sets were adopted for all the atoms, and the polarisation continuum model with the ethanol parameter and dielectric constant $(\varepsilon=24.6)$ was used to implicitly include solvent effects in the calculations. Formation energies were obtained by determining the energy difference between the sum of the energies of individual species and the energies of coordinated species. Natural bond orbital analysis was employed to evaluate the charge variation. IR vibration modes were obtained from frequency calculations, and the frequencies were scaled by 0.98 to match the experimental data.

\section{Data availability}

The data that support the findings of this study are available from the corresponding author upon reasonable request.

Received: 10 September 2020; Accepted: 16 March 2021; Published online: 22 April 2021

\section{References}

1. Choi, J. W. \& Aurbach, D. Promise and reality of post-lithium-ion batteries with high energy densities. Nat. Rev. Mater. 1, 16013 (2016).

2. Canepa, P. et al. Odyssey of multivalent cathode materials: open questions and future challenges. Chem. Rev. 117, 4287-4341 (2017).

3. Olivetti, E. A., Ceder, G., Gaustad, G. G. \& Fu, X. Lithium-ion battery supply chain considerations: analysis of potential bottlenecks in critical metals. Joule 1, 229-243 (2017).

4. Kwade, A. et al. Current status and challenges for automotive battery production technologies. Nat. Energy 3, 290-300 (2018).

5. Elia, G. A. et al. An overview and future perspectives of aluminum batteries. Adv. Mater. 28, 7564-7579 (2016).

6. Liang, Y., Dong, H., Aurbach, D. \& Yao, Y. Current status and future directions of multivalent metal-ion batteries. Nat. Energy 5, 646-656 (2020).

7. Yoo, D.-J., Kim, J.-S., Shin, J., Kim, K. J. \& Choi, J. W. Stable performance of aluminum-metal battery by incorporating lithium-ion chemistry. ChemElectroChem 4, 2345-2351 (2017).

8. Wen, X. et al. Materials compatibility in rechargeable aluminum batteries: chemical and electrochemical properties between vanadium pentoxide and chloroaluminate ionic liquids. Chem. Mater. 31, 7238-7247 (2019).

9. Dambournet, D. et al. Atomic insights into aluminium-ion insertion in defective anatase for batteries. Angew. Chem. Int. Ed. 59, 19247-19253 (2020).

10. Walter, M., Kravchyk, K. V., Böfer, C., Widmer, R. \& Kovalenko, M. V. Polypyrenes as high-performance cathode materials for aluminum batteries. Adv. Mater. 30, 1705644 (2018).

11. Wang, D.-Y. et al. Advanced rechargeable aluminium ion battery with a highquality natural graphite cathode. Nat. Commun. 8, 14283 (2017).
12. Yu, Z. et al. Flexible stable solid-state Al-ion batteries. Adv. Funct. Mater. 29, 1806799 (2019).

13. Yu, X., Wang, B., Gong, D., Xu, Z. \& Lu, B. Graphene nanoribbons on highly porous $3 \mathrm{D}$ graphene for high-capacity and ultrastable Al-ion batteries. Adv. Mater. 29, 1604118 (2017).

14. $\mathrm{Hu}, \mathrm{Y}$. et al. An innovative freeze-dried reduced graphene oxide supported $\mathrm{SnS}_{2}$ cathode active material for aluminum-ion batteries. Adv. Mater. 29, 1606132 (2017)

15. Hu, Y. et al. A binder-free and free-standing cobalt sulfide@carbon nanotube cathode material for aluminum-ion batteries. Adv. Mater. 30, 1703824 (2018).

16. Liang, K., Ju, L., Koul, S., Kushima, A. \& Yang, Y. Self-supported tin sulfide porous films for flexible aluminum-ion batteries. Adv. Energy Mater. 9, 1802543 (2019).

17. Cai, T. et al. Stable $\mathrm{CoSe}_{2} /$ carbon nanodice@reduced graphene oxide composites for high-performance rechargeable aluminum-ion batteries. Energy Environ. Sci. 11, 2341-2347 (2018).

18. Jiang, J. et al. One-dimensional $\mathrm{Cu}_{2-\mathrm{x}} \mathrm{Se}$ nanorods as the cathode material for high-performance aluminum-ion battery. ACS Appl. Mater. Interfaces 10, 17942-17949 (2018).

19. Lin, M.-C. et al. An ultrafast rechargeable aluminium-ion battery. Nature $\mathbf{5 2 0}$, 324-328 (2015)

20. Kaveevivitchai, W., Huq, A., Wang, S., Park, M. J. \& Manthiram, A. Rechargeable aluminum-ion batteries based on an open-tunnel framework. Small 13, 1701296 (2017).

21. Koketsu, T. et al. Reversible magnesium and aluminium ions insertion in cation-deficient anatase $\mathrm{TiO}_{2}$. Nat. Mater. 16, 1142-1148 (2017).

22. Xiong, P. et al. Strain engineering of two-dimensional multilayered heterostructures for beyond-lithium-based rechargeable batteries. Nat. Commun. 11, 3297 (2020)

23. Wang, S. et al. High-performance aluminum-ion battery with CuS@C microsphere composite cathode. ACS Nano 11, 469-477 (2017).

24. Yang, H. et al. An aluminum-sulfur battery with a fast kinetic response Angew. Chem. Int. Ed. 57, 1898-1902 (2018).

25. Yu, Z. et al. Hexagonal NiS nanobelts as advanced cathode materials for rechargeable Al-ion batteries. Chem. Commun. 52, 10427-10430 (2016).

26. Gao, T. et al. A rechargeable $\mathrm{Al} / \mathrm{S}$ battery with an ionic-liquid electrolyte. Angew. Chem. Int. Ed. 55, 9898-9901 (2016).

27. Jadhav, A. L., Xu, J. H. \& Messinger, R. J. Quantitative molecular-level understanding of electrochemical aluminum-ion intercalation into a crystalline battery electrode. ACS Energy Lett. 5, 2842-2848 (2020).

28. Geng, L., Lv, G., Xing, X. \& Guo, J. Reversible electrochemical intercalation of aluminum in $\mathrm{Mo}_{6} \mathrm{~S}_{8}$. Chem. Mater. 27, 4926-4929 (2015).

29. Geng, L. et al. Crystal structure transformation in chevrel phase $\mathrm{Mo}_{6} \mathrm{~S}_{8}$ induced by aluminum intercalation. Chem. Mater. 30, 8420-8425 (2018).

30. Bitenc, J. et al. Concept and electrochemical mechanism of an $\mathrm{Al}$ metal anode -organic cathode battery. Energy Storage Mater. 24, 379-383 (2020).

31. Fan, X. et al. A universal organic cathode for ultrafast lithium and multivalent metal batteries. Angew. Chem. Int. Ed. 57, 7146-7150 (2018).

32. Kim, D. J. et al. Rechargeable aluminium organic batteries. Nat. Energy 4 , 51-59 (2019)

33. Wang, S., Huang, S., Yao, M., Zhang, Y. \& Niu, Z. Engineering active sites of polyaniline for $\mathrm{AlCl}_{2}{ }^{+}$storage in an aluminum-ion battery. Angew. Chem. Int Ed. 59, 11800-11807 (2020).

34. Zhou, J., Yu, X., Zhou, J. \& Lu, B. Polyimide/metal-organic framework hybrid for high performance Al-organic battery. Energy Storage Mater. 31, 58-63 (2020).

35. Ji, Q. et al. Cyclotetrabenzoin: facile synthesis of a shape-persistent molecular square and its assembly into hydrogen-bonded nanotubes. Chem. Eur. J. 21, 17205-17209 (2015).

36. Hahn, S. et al. Synthesis and characterization of heterobenzenacyclooctaphanes derived from cyclotetrabenzoin. Chem. Eur. J. 23, 10543-10550 (2017).

37. Yoo, D.-J. \& Choi, J. W. Elucidating the extraordinary rate and cycling performance of phenanthrenequinone in aluminum-complex-ion batteries. $J$. Phys. Chem. Lett. 11, 2384-2392 (2020).

38. Lee, M. et al. Organic nanohybrids for fast and sustainable energy storage. Adv. Mater. 26, 2558-2565 (2014).

39. Song, Z. et al. Polyanthraquinone as a reliable organic electrode for stable and fast lithium storage. Angew. Chem. Int. Ed. 54, 13947-13951 (2015).

40. Li, K. et al. Evolution of the electrochemical interface in sodium ion batteries with ether electrolytes. Nat. Commun. 10, 725 (2019).

41. Wang, J., Polleux, J., Lim, J. \& Dunn, B. Pseudocapacitive contributions to electrochemical energy storage in $\mathrm{TiO}_{2}$ (Anatase) nanoparticles. J. Phys. Chem. C 111, 14925-14931 (2007).

42. Kohn, W. \& Sham, L. J. Self-consistent equations including exchange and correlation effects. Phys. Rev. 140, A1133-A1138 (1965). 


\section{Acknowledgements}

F.G. acknowledges funding from the European Union's Horizon 2020 research and innovation programme under the Marie Skłodowska-Curie grant agreement No 796024. J.W.C. acknowledges the support by the National Research Foundation of Korea (NRF) grants (NRF-2021R1A2B5B03001956, NRF-2017M1A2A2044504, NRF2020M3H4A3081874, and NRF-2018M1A2A2063340), the Technology Innovation Programme (20012341) funded by the Ministry of Trade, Industry \& Energy (MOTIE) of Korea and generous support from the Institute of Engineering Research (IOER) and Inter-university Semiconductor Research Center (ISRC) at Seoul National University. M.H. thanks the Royal Society and the Wolfson Foundation for support.

\section{Author contributions}

D.-J.Y., M.H., F.G. and J.W.C. designed the concept. F.G. synthesised and characterised the molecules. D.-J.Y. performed the electrochemical measurements, ex situ electrode analyses, and DFT calculations. D.-J.Y., M.H., F.G. and J.W.C. wrote the paper. F.G. and J.W.C. directed the research. All authors discussed the results and commented on the paper.

\section{Competing interests}

The authors declare no competing interests.

\section{Additional information}

Supplementary information The online version contains supplementary material available at https://doi.org/10.1038/s41467-021-22633-y.
Correspondence and requests for materials should be addressed to F.Göc. or J.W.C.

Peer review information Nature Communications thanks Guanjie He and the other anonymous, reviewer(s) for their contribution to the peer review of this work.

Reprints and permission information is available at http://www.nature.com/reprints

Publisher's note Springer Nature remains neutral with regard to jurisdictional claims in published maps and institutional affiliations.

(c) (i) Open Access This article is licensed under a Creative Commons Attribution 4.0 International License, which permits use, sharing, adaptation, distribution and reproduction in any medium or format, as long as you give appropriate credit to the original author(s) and the source, provide a link to the Creative Commons license, and indicate if changes were made. The images or other third party material in this article are included in the article's Creative Commons license, unless indicated otherwise in a credit line to the material. If material is not included in the article's Creative Commons license and your intended use is not permitted by statutory regulation or exceeds the permitted use, you will need to obtain permission directly from the copyright holder. To view a copy of this license, visit http://creativecommons.org/ licenses/by/4.0/.

(C) The Author(s) 2021 